\title{
PARTICLE-IN-CELL SIMULATIONS OF HALO PARTICLE PRODUCTION IN INTENSE CHARGED PARTICLE BEAMS PROPAGATING THROUGH A QUADRUPOLE FOCUSING FIELD WITH VARYING LATTICE AMPLITUDE*
}

\author{
Mikhail Dorf, Ronald C. Davidson and Edward Startsev \\ Plasma Physics Laboratory, Princeton University, Princeton, New Jersey, USA 08543
}

\begin{abstract}
The transverse compression and dynamics of intense charged particle beams, propagating through a periodic quadrupole lattice, play an important role in many accelerator physics applications. Typically, the compression can be achieved by means of increasing the focusing strength of the lattice along the beam propagation direction. However, beam propagation through the lattice transition region inevitably leads to a certain level of beam mismatch and halo formation. In this paper we present a detailed analysis of these phenomena using particle-in-cell (PIC) numerical simulations performed with the WARP code. A new definition of beam halo is proposed in this work that provides the opportunity to carry out a quantitative analysis of halo production by a beam mismatch.
\end{abstract}

\section{INTRODUCTION}

Alternating-gradient accelerators and transport systems have a wide range of applications ranging from basic scientific research to industrial applications [1]. Of considerable practical importance for several applications is the transverse focusing of the beam that can be achieved by means of increasing the focusing field strength of the alternating-gradient lattice along the beam propagation direction. It is evident that high-intensity charge bunch propagation through the lattice transition region inevitably leads to a certain level of beam mismatch and emittance growth. Furthermore, it is well known that a beam mismatch can produce halo particles that have much higher transverse energy than the core particles and may cause a degradation of beam quality. It is evident that the beam mismatch will decrease as the length of the transition region is increased, assuming that the lattice amplitude is constant outside the transition region. Therefore, it is a matter of considerable practical interest to determine how smooth (adiabatic) the lattice transition should be to assure that matching is maintained during the compression.

Transverse beam compression has recently been investigated by means of numerical simulations [2] and in the Paul Trap Simulator Experiment (PTSX) [3]. Although an extensive qualitative description of the halo formation process is given in [2], in the present paper more emphasis is placed on providing a quantitative treatment of the halo formation phenomena. The problem

\footnotetext{
*Research supported by the U.S. Department of Energy.
}

of quantitatively defining the beam halo was previously considered in $[2,4,5]$. In [4] a method for calculating dimensionless halo parameters based upon moments of the beam distribution function was proposed. These halo parameters can be used for comparing the 'halo intensity' for different beam distributions. However, no guidelines were provided on how to quantitatively distinguish a halo particle from a core particle, and hence the actual 'halo fraction' of all beam particles for a given distribution can not be estimated. Attempts to distinguish halo particles from the beam core particles were also made in $[2,5]$. However, these studies were based on a "visual analysis" of the transverse phase-spaces, and no rigorous mathematical criteria were applied. In the present work a more rigorous definition of halo particles is proposed, allowing one to estimate the actual number of halo particles produced by the beam mismatch.

\section{TIME EVOLUTION OF THE BEAM ENVELOPE AND TRANSVERSE EMITTANCE DURING COMPRESSION}

In this section we present a detailed numerical analysis of the evolution of low-order moments of the distribution function: the rms envelope dimensions and the transverse emittance. The simulations were performed using the two-dimensional transverse slice model based on the WARP electrostatic particle-in-cell (PIC) code [6], describing the beam response to an alternating-gradient quadrupole focusing field with varying lattice amplitude. To model the lattice we assume the sinusoidal lattice function, $\mathrm{k}_{\mathrm{q}}(\mathrm{z})=\mathrm{A}(\mathrm{z}) \cdot \sin \left(\mathrm{z} / \mathrm{S}_{\mathrm{L}}\right)$, where $\mathrm{S}_{\mathrm{L}}$ is the lattice period (length of a single focusing cell), and $\mathrm{A}(\mathrm{z})$ is the function describing the variation of the lattice amplitude. To describe the compression, we adopt a simple model in which $\mathrm{A}(\mathrm{z})$ varies according to

$$
A(z)=A_{i}+\left(A_{f}-A_{i}\right) \cdot\left[\exp \left(4\left(z_{1 / 2}-z\right) / L_{t r}\right)+1\right]^{-1},(1)
$$

where $\mathrm{z}_{1 / 2}$ corresponds to the center of the transition region, and $\mathrm{L}_{\mathrm{tr}}$ is the characteristic length scale for the variation of $A(z)$ from the constant value $A_{i}$ to the constant value $A_{f}=2 A_{i}$. The evolution of the normalized rms envelope $\mathrm{x}$-dimension, $\mathrm{X}_{\mathrm{rms}}$, during compression for the cases of a space-charge-dominated beam and a moderate intensity beam is presented in Fig. 1. Note that Fig. 1 illustrates the $X_{\text {rms }}$ values calculated at the end of each focusing cell, and such a graphical representation for a matched beam would be a constant line. During the transition, the lattice strength measured by the vacuum 
phase advance [1] is changing from $\sigma_{i}^{\text {vac }}=17.4^{\circ}$ to $\sigma_{i}{ }^{\mathrm{vac}}=35.3^{\circ}$. The initial and final ratios of the depressed phase advance to the vacuum phase advance $\sigma / \sigma_{\text {vac }}$ [1] for the case of adiabatic compression is shown in Fig. 1. An important qualitative difference is evident when comparing the evolution of the beam mismatch caused by a nonadiabatic compression for different beam intensities. Strong beam mismatch oscillations are only slightly damped for a space-charge-dominated beam and almost completely damped for a beam with moderate intensity. A plausible explanation of this
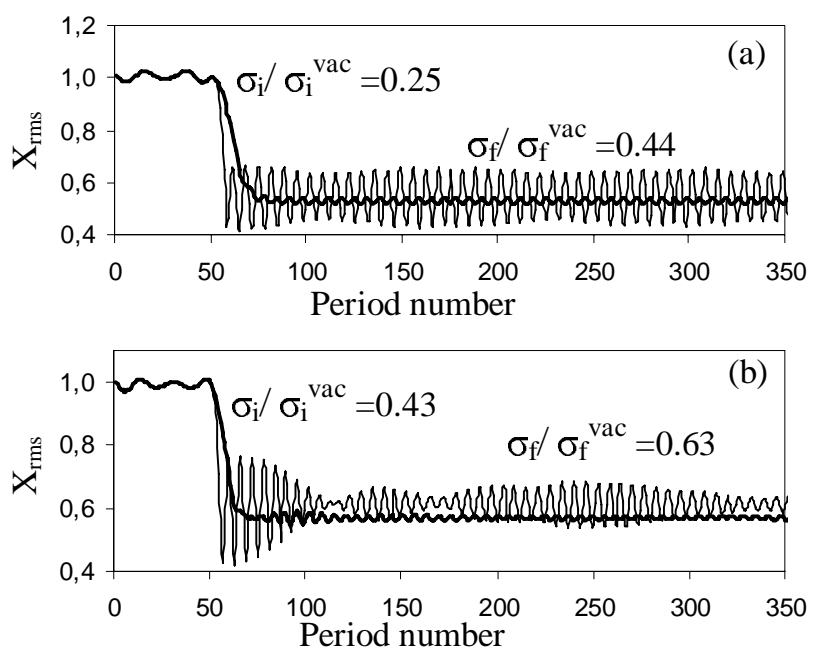

Figure 1: Rms envelope $\mathrm{x}$-dimension verses number of lattice periods. (a) Adiabatic compression with $\mathrm{L}_{\mathrm{tr}} / \mathrm{S}_{\mathrm{L}}=15$ (bold line) and nonadiabatic compression with $\mathrm{L}_{t \mathrm{r}} / \mathrm{S}_{\mathrm{L}}=6$ (fine line) for a space-charge-dominated beam. (b) Adiabatic compression with $\mathrm{L}_{\mathrm{tr}} / \mathrm{S}_{\mathrm{L}}=10$ (bold line) and nonadiabatic compression with $\mathrm{L}_{\mathrm{tr}} / \mathrm{S}_{\mathrm{L}}=4$ (fine line) for a beam with moderate intensity.

phenomena is proposed in [7], where the beam particles are considered as an ensemble of betatron oscillators coupled to the collective mismatch envelope oscillations. The damping occurs due to the energy transfer from the collective oscillations to the oscillators (beam particles), which are close to parametric (fundamental 2:1) resonance with the mismatch oscillations. In the same work [7] it was shown that for the case of a space-chargedominated beam most of the betatron oscillators are far from the parametric resonance, providing a slight damping of the collective oscillations. As the beam spacecharge intensity decreases, the mismatch frequency approaches the frequency distribution of the betatron oscillators, providing an increased mixing of the collective oscillations.

The relaxation process described above transfers energy from the collective oscillations to the transverse motion of the resonant particles, thereby increasing the transverse phase-space area (emittance growth). The simulations demonstrate that the emittance variations in the transition region is negligible compared to the subsequent growth during the mismatch relaxation. Figure 2 shows the normalized emittance value at the final stage of compression, when the mismatch relaxation process is completed, for different characteristic lengths of the transition region.

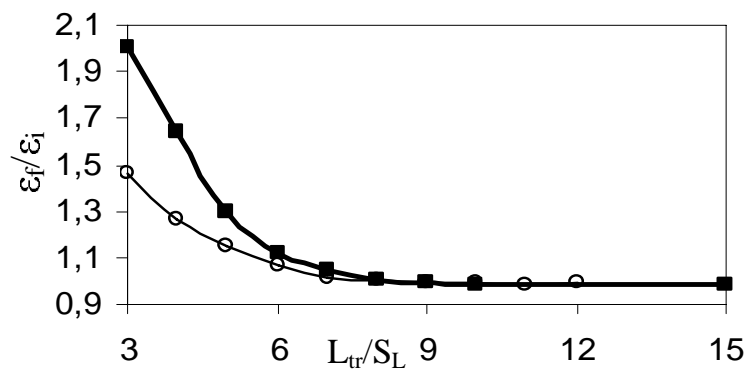

Figure 2: Ratio of the final to initial beam emittance versus characteristic transition length, $\mathrm{L}_{\mathrm{tr}}$. Circles correspond to $\sigma_{\mathrm{i}} / \sigma_{\mathrm{i}}^{\mathrm{vac}}=0.43$, and squares to $\sigma_{\mathrm{i}} / \sigma_{\mathrm{i}}^{\mathrm{vac}}=0.25$.

\section{HALO FORMATION DURING THE COMPRESSION PROCESS}

In this section, we present an analysis of the halo formation during the compression process using the WARP code for an alternating-gradient quadrupole lattice. Figure 3 illustrates the final $\left(\mathrm{X} ; \mathrm{X}^{\prime}\right)$ phase spaces for adiabatic and non-adiabatic compression. The scaled coordinates $\mathrm{X}=\mathrm{x} /\left(2 \cdot \mathrm{X}_{\mathrm{rms}}\right)$ and $\mathrm{X}^{\prime}=2\left(\mathrm{X}_{\mathrm{rms}} \mathrm{X}^{\prime}-\mathrm{X}_{\mathrm{rms}}^{\prime} \mathrm{x}\right) / \varepsilon_{\mathrm{x}}$ are plotted to remove the envelope oscillations. Here, prime denotes derivative with respect to $z$. Note, that the $2: 1$ resonance points are located on the $\mathrm{X}$ axis when the core radius is a minimum, and on the $\mathrm{X}^{\prime}$ axis when the core radius is a maximum.
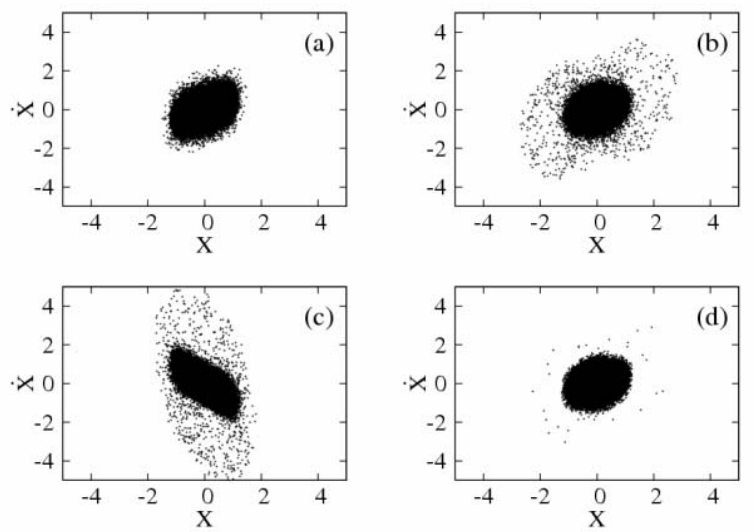

Figure 3: Plots of the instantaneous $\left(X ; X^{\prime}\right)$ phase space for a space-charge-dominated beam with $\sigma_{\mathrm{i}} / \sigma_{\mathrm{i}}^{\mathrm{vac}}=0.25$ : (a) final stage of adiabatic compression with $\mathrm{L}_{\mathrm{tr}} / \mathrm{S}_{\mathrm{L}}=15$; (b) and (c) final stage of nonadiabatic compression with $\mathrm{L}_{\mathrm{tr}} / \mathrm{S}_{\mathrm{L}}=6$ corresponding to the minimum and maximum beam radius, respectively; (d) plot of the phase-space presented in case (b) after removing particles with $\omega_{\beta}>\omega_{\text {odd }} / 2$.

We emphasize here some interesting features of the halo formation. The simulations show that during the transition stage only a small number of particles leave the core, whereas most particles populate the halo region during the relaxation stage. Furthermore, it is found that 
the halo formation process saturates along with the damping of the mismatch mode. These details are evidence that the main mechanism for halo formation is indeed an energy transfer from the collective modes to the resonant particles (halo particles) during the parametric resonance interaction.

It is of considerable practical interest to estimate the actual number of halo particles produced by a beam mismatch. Previous quantitative studies of beam halos did not provide a criterion for distinguishing a halo particle from a core particle, and therefore cannot be applied to estimate the number of halo particles in a given distribution. In this paper we propose a simple quantitative definition of a halo particle based on an analysis of the beam betatron frequency distribution.

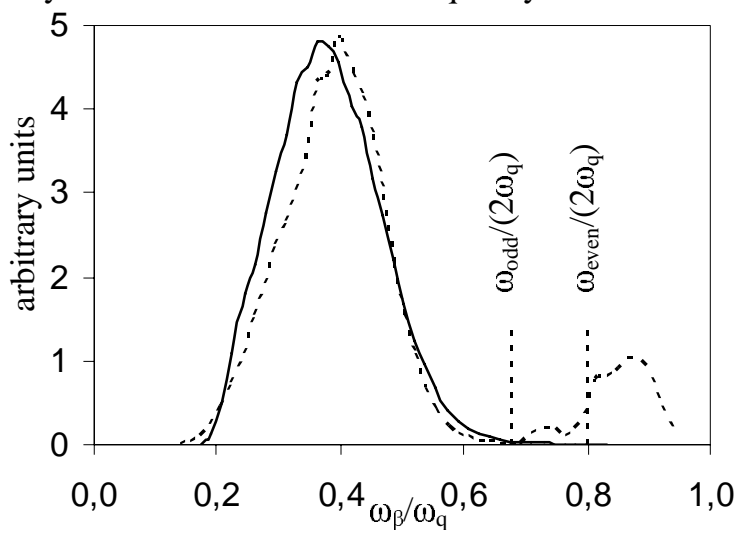

Figure 4: Beam betatron frequency distribution at the final stage of adiabatic compression with $\mathrm{L}_{\mathrm{tr}} / \mathrm{S}_{\mathrm{L}}=15$ (solid line), and nonadiabatic compression with $\mathrm{L}_{\mathrm{tr}} / \mathrm{S}_{\mathrm{L}}=3$ (broken line) for a space-charge-dominated beam with $\sigma_{\mathrm{i}} / \sigma_{\mathrm{i}}^{\mathrm{vac}}=0.25$. Here, $\omega_{\mathrm{q}}$ is the smooth-focusing frequency for the final state. Envelope frequencies $\left(\omega_{\text {odd }}\right.$ and $\left.\omega_{\text {even }}\right)$ are calculated within the smooth-focusing model.

Figure 4 shows the beam betatron frequency distribution at the final stage of compression. For the case of adiabatic compression, the distribution function is bellshaped with an exponentially decaying tail. However, for nonadiabatic compression a "bump-on-tail" structure appears. It is natural to assume that particles with high betatron frequency forming this bump structure are halo particles. Indeed, it is well know that the energydependent betatron frequency is much higher for energetic halo particles than for core particles. Moreover, the non-monotonic tail structure is represented by the two bumps corresponding to half-values of the even (symmetric), $\omega_{\text {even, }}$ and the odd (quadrupole) $\omega_{\text {odd }}$ envelope mode frequencies (see Fig.4). Also, the parametric resonance interaction between the halo particles and the collective oscillations (mismatch modes) provides an increase in particles energy and betatron frequency. Therefore, at the final stage the bumps should be located to the right of the half-value of the minimum envelope mode frequency, which is the odd mode frequency. Indeed, Fig. 4 (for nonadiabatic compression) demonstrates this feature. Finally, Fig. 3(d) shows the X-
$\mathrm{X}^{\prime}$ phase space after removing particles with betatron frequency higher than the odd envelope frequency halfvalue, $\omega_{\beta}>\omega_{\text {odd }} / 2$. The beam core is now surrounded by only a few halo particles, which are still present because the energy transfer process is not yet completed. We may now formulate the following quantitative definition of a halo particle. If the particle betatron frequency is greater than the odd envelope frequency half-value then it is a halo particle. It should be noted, that according to this definition even a matched beam with a thermal equilibrium distribution function has a certain fraction of halo particles. This fraction is exponentially small for a space-charge-dominated beam, but it increases with decreasing beam intensity since the mismatch frequency approaches the frequency distribution of the betatron oscillators. Figure 5 shows the halo fraction of all the simulation particles at the final stage of compression for different lengths of the transition region.

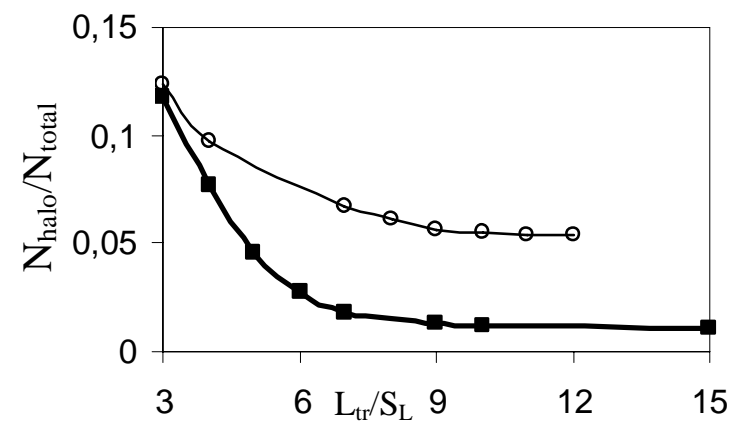

Figure 5: Halo fraction of all simulation particles versus characteristic transition length, $\mathrm{L}_{\mathrm{tr}}$. Circles correspond to $\sigma_{\mathrm{i}} / \sigma_{\mathrm{i}}^{\mathrm{vac}}=0.43$, and squares to $\sigma_{\mathrm{i}} / \sigma_{\mathrm{i}}^{\mathrm{vac}}=0.25$.

\section{CONCLUSIONS}

The halo formation process during transverse beam compression has been investigated by means of numerical simulations using the WARP PIC code, describing the beam response to an alternating-gradient quadrupole focusing field with varying lattice amplitude. A new quantitative definition of halo particles proposed in this paper allowed us to estimate the number of halo particles produced by a beam mismatch.

\section{REFERENCES}

[1] R. C. Davidson and H. Qin, Physics of Intense Charged Particle Beams in High Energy Accelerators (World Scientific, Singapore, 2001), and references therein.

[2] M.Dorf et al, Phys. Rev. ST Acel. Beams 9, 034202 (2006).

[3] M.Chung et al, Phys. Rev. ST Accel. Beams 10, submitted for publication (2007).

[4] C. K. Allen, T. P.Wangler Rev. ST Accel. Beams 5, 124202 (2002).

[5] H. Okamoto, M. Ikegami, Phys. Rev. E 55, 4694 (1997).

[6] D. P. Grote et al, Nucl. Instrum. Methods Phys. Res. A 415, 428 (1998).

[7] V. Variale, Phys. Rev. ST Accel. beams 4, 084201 (2001). 